\title{
Corticobasal Degeneration Misdiagnosed As Musculoskeletal Disease: A Challenging Diagnosis of Higher-Level Gait Disease
}

\author{
Halil Onder ${ }^{1}$ \\ 1Department of Neurology, Yozgat City Hospital, Yozgat, Turkey
}

\author{
Address for correspondence Halil Onder, MD, Department of \\ Neurology, Yozgat City Hospital, Yozgat, Turkey \\ (e-mail: halilnder@yahoo.com).
}

\begin{abstract}
Keywords

- corticobasal degeneration

- higher level gait

disease

- misdiagnosis

Gait disorders are common in the elderly as there are various causes of neurological and non-neurological conditions. On the other hand, most of the gait parameters do change with advancing age which is identified as age-related physiological changes in gait. At this point, the discrimination between age-related physiological changes and gait disorders may be strictly challenging. After identifying gait as an abnormal pattern, classification of it and making the responsible pathophysiology also require high-level expertise in this regard. Herein, we present a rare patient with corticobasal degeneration (CBD) who had admitted initially due to complaints of gait problems. Over a long time, the patient had received the misdiagnosis of gait abnormality due to musculoskeletal problems by multiple physicians. However, the detailed neurological exam showed a higher level gait disorder (HLGD). Further investigations at this point yielded the diagnosis of CBD.
\end{abstract}

\section{Introduction}

Gait disorders are common in the elderly population. The prevalence of gait disorders has been reported to be $10 \%$ in people aged 60 to 69 years and it may rise to $60 \%$ in community-dwelling subjects aged over 80 years. ${ }^{1}$ Among them, higher level gait disorders (HLGD) may be the most challenging one to be distinguished from normal gait pattern, and its specific diagnosis may be strictly hard as the awareness of this entity is insufficient among clinicians. ${ }^{2}$ In this report, we present a patient with a rare etiology of HLGD whose gait pattern had initially been diagnosed as musculoskeletal gait problems. We aim to draw attention to this rare subtype of gait disorder and suggest some crucial remarks to be kept in mind for avoiding diagnostic errors.

\section{Case Report}

A 61-year-old female patient had admitted due to walking difficulty and balance problems particularly while turnings which had started over the last 2 years. It has learned that she had visited multiple times to outpatient clinics of the orthopedics and traumatology, physical therapy, and rehabilitation and neurology departments within this time interval. However, no neurological disease was suspected; otherwise, the walking problem was associated with plantar fasciitis in her right leg and mild lumbar arthritis. Of note, the patient stated that temporary mild improvement in her gait was achieved by nonsteroidal anti-inflammatory drug therapy. However, due to the deterioration of gait, she had continued to admit to other physicians. The family medical history was unremarkable. On neurological examination at admission to our clinic, the patient was evaluated as orientated and cooperative. The examination of the eye movements revealed normal saccadic and pursuit eye movements. Motor, sensory, and cerebellar examinations were within normal limits. Deep tendon reflexes were bilateral normal. However, her gait was evaluated as mildly broad-based and she had difficulty particularly during turnings. Besides, the extrapyramidal examinations revealed mild contractions of the right distal lower limb during walking that was compatible with mild dystonia. Cranial magnetic resonance imaging (MRI) examination revealed significant atrophy in the left parietal

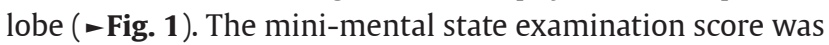
28 points. Hence, the patient was reinterrogated in light of the findings of parietal atrophy on MRI and neurological 

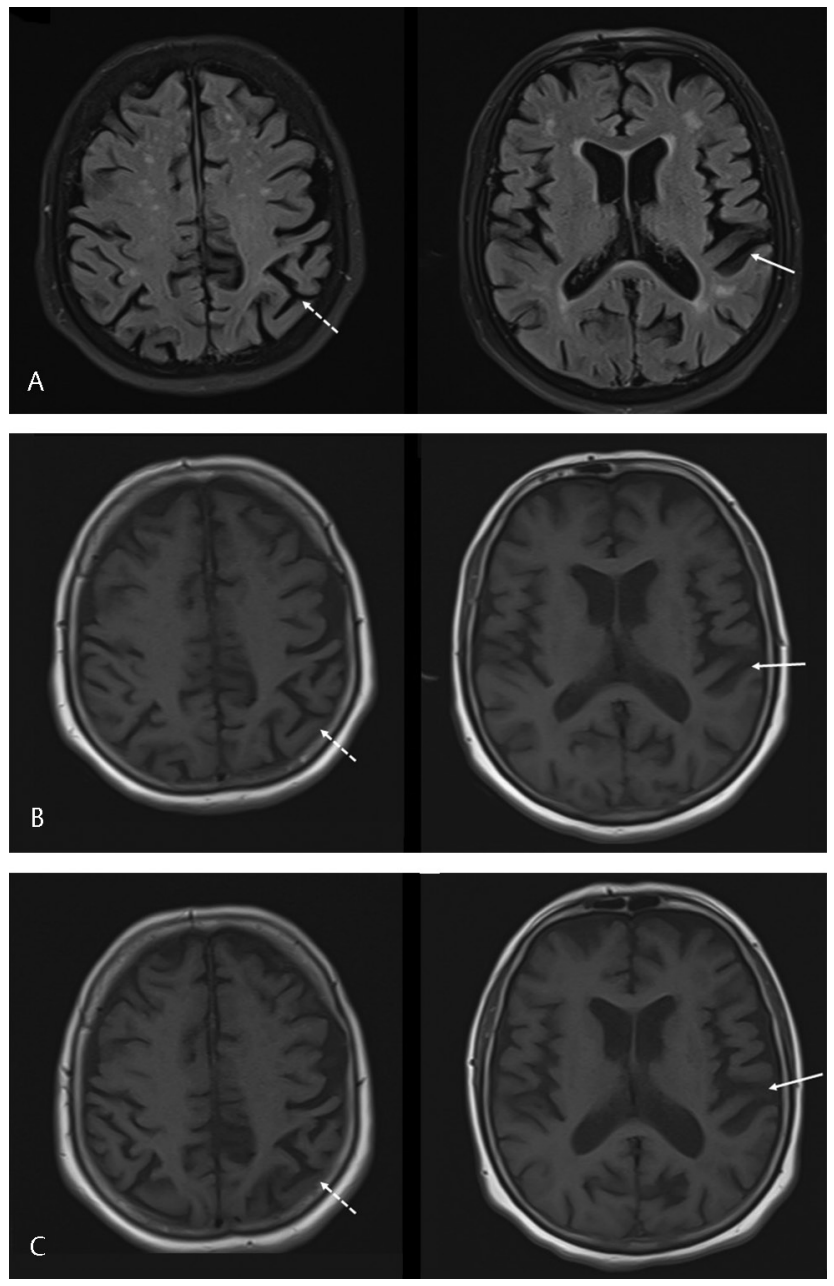

Fig. 1 Cranial magnetic resonance images showing atrophy in the left posterior parietal cortex (jagged arrows) and temporal cortex (arrows). (A) FLAIR images in June 2019. (B) T1 weighted images in June 2019. (C) FLAIR images in December 2019. FLAIR, fluid attenuated inversion recovery.

exam of cortical gait abnormality. She had not a history of restless leg syndrome or rapid eye movement sleep behavior disorder. Besides, she did not have symptoms of orthostatic hypotension, and the complaints of urinary incontinence and constipation were absent. However, arrestingly, the patient stated that she had been ignoring her right leg and suffered from a sensation of involuntary motor activity of her right leg in and the feeling of estrangement from that limb. This was terminologically compatible with the "alien limb phenomenon," which is known as a diagnostic feature of CBD. Besides, repeated detailed sensory investigations yielded stereognosis and graphesthesia in the right extremities showing the disturbances of cortical sensory functions. To demonstrate the perfusion dynamic, positron emission tomography (PET) was performed which showed severe hypoperfusion in the left posterior parietal cortex supporting the diagnosis of CBD (-Fig. 2). The patient and her relatives were informed about the disease and progress. Levodopa was tried (up to $3 \times 200 \mathrm{mg}$ ) which did not provide any amelioration in her symptoms. Remarkably, the clinic of the patient was found to deteriorate apparently at the polyclinic visit 12 months
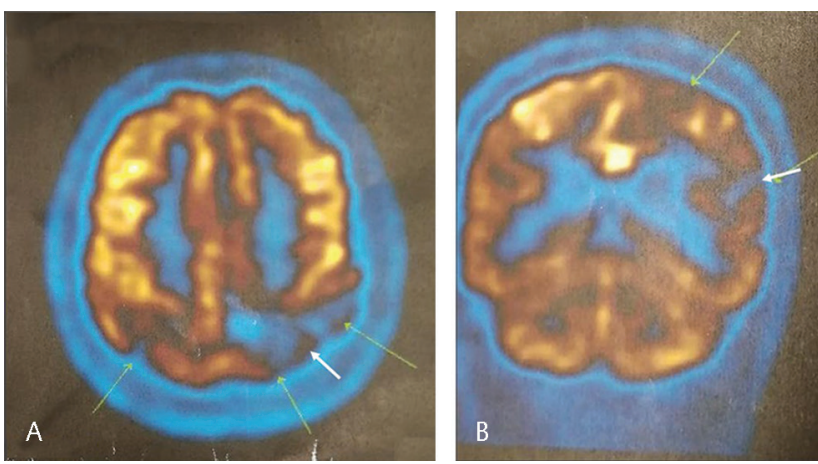

Fig. 2 Positron emission tomography images showing severe hypoperfusion in the left posterior parietal cortex. (A) Axial image. (B) Coronal image.

later. Neurological examination at this time showed that her speech was dysarthric and moderate right-sided paresis had developed in the interval period ( $4 / 5$ on MRC). Besides, extrapyramidal examinations showed right bradykinesia and dystonic posture in the right lower extremity ( - Video 1 ). The patient could not walk even short distances without support.

\section{Video 1}

Neurological examination showing bradykinesia more prominent on the right side and right-sided mild paralysis. Online content including video sequences viewable at: https://www.thieme-connect.com/products/ejournals/ html/10.1055/s-0040-1716773.

\section{Discussion}

In this report, we present a rare patient with CBD at whom the diagnosis was challenging and may provide substantial clinical remarks to be kept in mind. Initially, the patient had been suffering from mild gait difficulty and balance problems. However, no paralysis or cerebellar ataxia was determined, and the remarkable point was that the gait difficulty had been associated with musculoskeletal system problems again and again by several clinicians (including neurologists) previously. A big part of the gait disorders in the elderly subjects belongs to non-neurological causes including hip and knee osteoarthritis. ${ }^{3}$ Besides, most of the gait parameters such as gait velocity, cadence, walking posture, and joint motion do change with advancing age, which is identified as age-related physiological changes in gait. Taken together, the differentiation of an abnormal gait pattern of neurological origin from the physiological gait as well as identification of the abnormal gait and subgrouping it may be strictly challenging in the elderly population. In this patient, the gait problem can be classified under the head of HLGD. The HLGD is a term used to define locomotor and balance difficulties, which cannot be explained by peripheral motor, sensory, pyramidal, cerebellar, or basal ganglia lesions. ${ }^{2}$ Actually, HLGD is a category of gait disorders and incorporate all the clinical gait and 
balance disorders that cannot be explained by the classic motor or sensory deficits resulting in impairments of gait and balance. Regarding the etiological aspects, [removed hyperlink field] HLGD is not a single entity; on the contrary, there may be a variety of neurodegenerative, vascular, neoplastic conditions, etc. that may lead to HLGD. However, the awareness of this entity is the critical stage for the proper diagnosis as the most challenging subtype of gait disease is HLGD. The remarkable point is that HLGD does not include neurological signs such as weakness, sensory deficits, rigidity,bradykinesia, ataxia, spasticity, or hyporeflexia why the diagnosis strictly perplex the clinicians. ${ }^{2}$ In our patient, the initial and challenging stage was the identification of the underlying HLGD which led us to seek etiological investigations and make the final diagnosis of CBD. At this stage, the clinical manifestation of mild broad-based gait, hesitations during turnings, and postural disturbances were crucial clues.

Clinically patients with CBD usually manifest with an asymmetric motor disorder of the upper limb as well as accompanying myoclonic jerks and severe apraxia. The lower limbs may be involved but usually asymmetrically. However, isolated gait disturbance has rarely been reported as the initial manifestation of CBD. ${ }^{4}$ Gait abnormalities (often poorly characterized) were described in $73 \%$ during the entire course of the disease in CBD, but only 33\% at the onset, with postural instability and falls occurring at similar frequencies. ${ }^{5}$ The gait abnormality in the initial phase was reported to be present in $29 \%$ of the patients in another cohort study. ${ }^{6}$ Neurological examination of the patient at initial admission to our clinic revealed mild lower extremity dystonia plus cortical sensory deficit and alien limb phenomena leading the diagnosis of possible CBD according to the Armstrong criteria. ${ }^{5}$ At this time, PET findings also supported the diagnosis of CBD. However, on the last follow-up 12 months later, extrapyramidal abnormalities including significant right-sided bradykinesia and rigidity were involved in the clinic, and the limb dystonia was more prominent supporting the diagnosis of probable CBD. ${ }^{5}$

In conclusion, herein, we illustrate an informative patient with CBD who had presented with gait impairment in the initial phase. Based on this remarkable illustration, first, we emphasize the importance of the proper evaluation of the gait disturbance and recognition of HLGD among physicians to avoid underdiagnosis of crucial neurological diseases. Second, we point out CBD as a rare cause of isolated HLDS which may constitute a strictly challenging diagnosis particularly at early phases of the disease.

\section{Funding}

None.

\section{Conflict of Interest}

None declared.

\section{References}

1 Mahlknecht P, Kiechl S, Bloem BR, et al. Prevalence and burden of gait disorders in elderly men and women aged 60-97 years: a population-based study. PLoS One 2013;8(7):e69627

2 Nutt JG. Higher-level gait disorders: an open frontier. Mov Disord 2013;28(11):1560-1565

3 Pirker W, Katzenschlager R. Gait disorders in adults and the elderly : a clinical guide. Wien Klin Wochenschr 2017;129(3-4): 81-95

4 Rossor MN, Tyrrell PJ, Warrington EK, Thompson PD, Marsden CD, Lantos P. Progressive frontal gait disturbance with atypical Alzheimer's disease and corticobasal degeneration. J Neurol Neurosurg Psychiatry 1999;67(3):345-352

5 Armstrong MJ, Litvan I, Lang AE, et al. Criteria for the diagnosis of corticobasal degeneration. Neurology 2013;80(5):496-503

6 Alexander SK, Rittman T, Xuereb JH, Bak TH, Hodges JR, Rowe JB. Validation of the new consensus criteria for the diagnosis of corticobasal degeneration. J Neurol Neurosurg Psychiatry 2014;85(8):925-929 